\title{
Improvement of Light Extraction Efficiency of LED Packages Using an Enhanced Encapsulant Design
}

\author{
Hyun-Su Choi ${ }^{1}$, Joon-Sik Park², and Cheol-Hee Moon ${ }^{1 *}$ \\ ${ }^{1}$ Department of Display Engineering, Hoseo University, Asan 336-795, Korea \\ ${ }^{2}$ Department of Materials Science \& Engineering, Hanbat National University, Daejeon 305-719, Korea
}

(Received March 31, 2014 : revised June 11, 2014 : accepted June 11, 2014)

\begin{abstract}
We optimized the design of the flat encapsulant of a light-emitting diode (LED) package to obtain higher light output power (LOP), both by experiment and simulation using three-dimensional ray-tracing software. In the experiment, the refractive index of the encapsulant was varied (1.41 and 1.53). In addition, double-layer structures with these refractive indices $(1.41 / 1.53)$ were investigated by varying the shape of the interface between the two among flat, concave, and convex. The experiments showed that the LOP of the double-layer encapsulant with convex interface increased by $13.4 \%$ compared to the single-layer encapsulant with a refractive index 1.41, which was explained by the increase of the light extraction efficiency (LEE) in connection with the increase of the critical angle $\left(\theta_{c}\right)$ and the decrease of the Fresnel reflection.
\end{abstract}

\footnotetext{
Keywords : Light-emitting diode (LED), Encapsulant, Light extraction efficiency (LEE), Fresnel reflection, 3-D ray-tracing simulation

OCIS codes : (230.0230) Optical devices; (230.3670) Light-emitting diodes; (260.0260) Physical optics; (260.6970) Total internal reflection
}

\section{INTRODUCTION}

Regardless of the advantages of light-emitting diodes (LEDs), including high energy efficiency, long lifetime, vivid colors, high reliability, environmental protection, and safety, the external quantum efficiency (EQE, $\left.\eta_{\text {ext }}\right)$ is still an important issue that must be addressed to meet practical needs $[1,2]$. The EQE of an LED can be expressed as the product of the current injection efficiency (CIE, $\eta_{\text {inj }}$ ), internal quantum efficiency (IQE, $\eta_{\text {int }}$ ), and light extraction efficiency (LEE, $\eta_{\mathrm{extr}}$ ) $[3,4]$. The LEE of an LED depends strongly on the critical angle $\theta_{\mathrm{c}}$ for total internal reflection at the interface between the two media, which is determined by Eq. (1) as follows:

$$
\theta_{c}=\sin ^{-1}\left(\frac{n_{2}}{n_{1}}\right)
$$

where $n_{1}$ and $n_{2}$ are the refractive indices of the media on the incident and transmitted sides respectively.
The LEE is defined as the fraction of photons generated in the active region of the semiconductor that escape into free space. As shown in Fig. 1, the LEE based on the light-escape cone from the LED chip can be determined with Eq. (2) using $\theta_{\mathrm{c}}$ :

$$
L E E_{\theta C}=\frac{\int_{0}^{\theta_{c}} 2 \pi \sin (\theta) d \theta}{\int_{0}^{\frac{\pi}{2}} 2 \pi \sin (\theta) d \theta}
$$

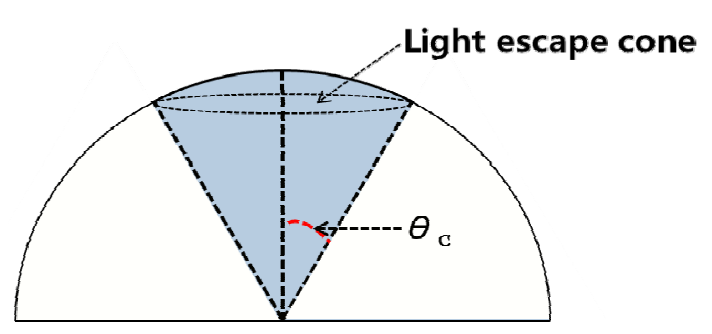

FIG. 1. Light extraction cone determined by the critical angle.

\footnotetext{
*Corresponding author: chmoon@hoseo.edu

Color versions of one or more of the figures in this paper are available online.
} 
Gallium nitride $(\mathrm{GaN})$ is most commonly used for LED chips, and emits blue light. In the case of the GaN/air interface, unfortunately, the refractive index of $\mathrm{GaN}$ is relatively large $(n=2.54)$ compared to that of air $(n=1)$. This large refractive index mismatch results in a narrow light-escape cone with $\theta_{\mathrm{c}}$ of $23.18^{\circ}$, as calculated with Eq. (1). Light impinging on the interface outside the extraction cone is subject to total internal reflection and eventually reabsorbed in the semiconductor or its metallic contacts $[5,6]$. A great amount of the light emission from quantum wells is lost within the GaN layer due to light confinement and subsequent reabsorption, which results in a temperature increase in the $p-n$ junction. Therefore, many methods have been proposed to improve the light extraction rate, including designs for the chip's structure [7], surface roughening [8], micropatterning[9], nanopatterning of the chip's surface [10], coatings of porous films and photonic crystals [11], and index matching [12].

Index matching via an intermediate layer between the LED chip and the air has been used to increase the LEE [13]. An encapsulant is a type of intermediate layer for index matching consisting of a phosphor and an epoxy to fix the phosphor. For encapsulants with intermediate refractive indices, $\theta_{c}$ increases and ultimately LEE increases. There have been many studies of encapsulants addressing modification of the shape of the encapsulation [14], addition of particles into the encapsulant [15], the effects of the hardening characteristics of the encapsulant [16], the development of encapsulants with high refractive indices [17, 18], and multilayered encapsulation [19]. Most of these studies have focused on spherical encapsulants, but because of the current trend of using high-power, multichip LED packages, the need to study flat encapsulants has arisen.

For this reason, this study is focused on how to increase the LEE of LED packages in which the top surface of the encapsulant is flat. In this study, the refractive index of the encapsulant was varied (1.41 and 1.53) and also doublelayer structures featuring the two indices $(1.41 / 1.53)$ were investigated by varying the shape of the interface between the two among flat, concave, and convex. We investigated the change in the light output according to the experimental conditions, from the viewpoint of the critical angle and Fresnel reflection. The optical properties of the LED package were measured using an integrating sphere to investigate the change in light output. The measured light output was compared to results simulated using three-dimensional (3-D) ray-tracing to ensure the validity of the new encapsulant design.

\section{EXPERIMENT}

\subsection{Constructing the LED Packages}

Table 1 shows the general specifications for each component of the single-chip LED package that was used in this study. A vertical GaN chip was used to obtain blue emission light, and was covered with a flat encapsulant as shown in Fig. 2.

Each LED chip was bonded to an $\mathrm{Al}_{2} \mathrm{O}_{3}$ submount (ceramic substrate) using $\mathrm{Ag}$ epoxy as a thermal interface material (TIM). The submount was made of low-temperature cofired ceramics (LTCCs) consisting mainly of alumina. Negative electrodes were located a top the LED chips and were electrically connected to the circuit lines using $\mathrm{Au}$ wire bonding. Positive electrodes $(\mathrm{Au} / \mathrm{Ni} / \mathrm{Cu}$ coated) were located on the bottoms of the LED chips and were electrically connected to the circuit pads on the upper surface of the submount using a TIM. 1.4-mm-thick ceramic ribs to protect the chip were attached to the substrate using ceramic paste. The encapsulant was dispensed to fill inside the ceramic package and form a flat top surface, and was hardened using a two-step heat treatment $\left(150^{\circ} \mathrm{C}\right.$ for 3 hours, followed by $200^{\circ} \mathrm{C}$ for 2 hours). In the case of the double-layer structure with two different encapsulant materials, the upper layer was dispensed after the lower layer had hardened. The refractive index of the LED chip was 2.54, while the refractive index of the encapsulant was varied using values of 1.41 and 1.53 .

TABLE 1. General specifications for each component of the LED package

\begin{tabular}{c|c|c|c}
\hline \hline & Material & Dimension $(\mathrm{mm})$ & $\begin{array}{c}\text { Refractive } \\
\text { index }\end{array}$ \\
\hline Chip & $\mathrm{GaN}$ & $1.016 \times 1.016 \times 0.18(\mathrm{t})$ & 2.54 \\
\hline Encapsulant & Silicone & $9 \times 9$ in bottom area & 1.41 and 1.53 \\
\hline Substrate & $\mathrm{Al}_{2} \mathrm{O}_{3}$ & $10 \times 10$ in bottom area & - \\
\hline MPCB & $\mathrm{Al}$ & $\Phi 28.5 \times 1.7(\mathrm{t})$ & - \\
\hline $\begin{array}{c}\text { Ceramic } \\
\text { wall }\end{array}$ & $\mathrm{Al}_{2} \mathrm{O}_{3}$ & $0.5 \times 10 \times 0.7(\mathrm{t})$ & - \\
\hline $\begin{array}{c}\text { Electrode } \\
\mathrm{Au} / \mathrm{Ni} / \mathrm{Cu} \\
(\text { coated })\end{array}$ & - & - \\
\hline TIM & $\mathrm{Ag} \mathrm{epoxy}$ & $1.016 \times 1.016 \times 0.01(\mathrm{t})$ &
\end{tabular}

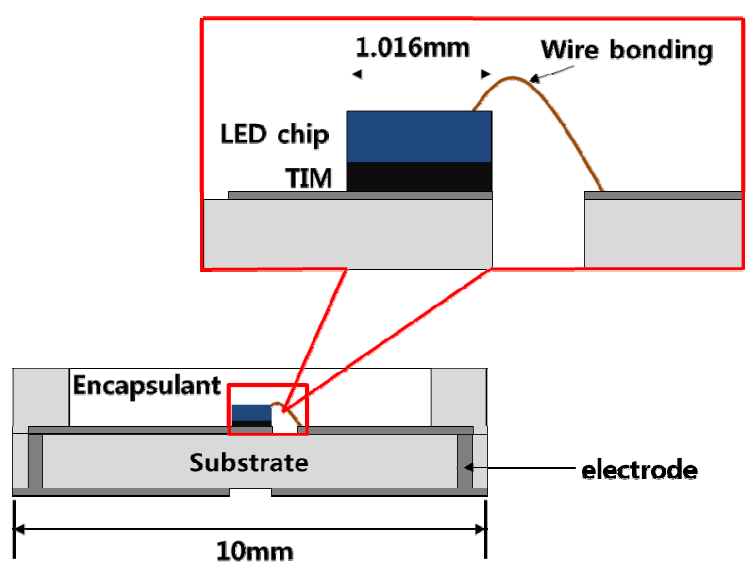

FIG. 2. Schematic drawing of the single-chip LED package used in this study. 


\subsection{Measurement of LOP of the LED Package}

We put the LED package into an integrating sphere $1 \mathrm{~m}$ in diameter to measure the LOP of the package. The light flux emitted from the package was scattered onto the inner surface of the integrating sphere and measured by the detector. The measurement was conducted under operating conditions of $350 \mathrm{~mA}$ and $3.2 \mathrm{~V}$.

\subsection{3-D Ray-tracing Simulations}

We used 3-D ray-tracing simulation software in which the light distribution and output were measured by detecting the number of the rays captured at the receiver. The light distribution was analyzed using ray-tracing simulation based on the Monte Carlo method and Snell's law.

Figure 3 is a schematic drawing of the LED chip, which consists of $\mathrm{Si}$ substrate, reflector layer, $p-\mathrm{GaN}, \mathrm{GaN}$, and $n$-GaN layers. GaN, an emission layer of the chip, has an absorption coefficient of $8 \mathrm{~mm}^{-1}$ and a refractive index of 2.54. The $p$-GaN and $n$-GaN layers have absorption coefficient of $5 \mathrm{~mm}^{-1}$ and refractive indices of 2.45 and 2.42 respectively [20]. It was assumed that in the reflector layer of the LED chip $50 \%$ of the incident rays were reflected, $30 \%$ scattered by Lambertian diffuse scattering, and $20 \%$ absorbed. It was also assumed that in the silicon $(\mathrm{Si})$ substrate $50 \%$ of rays were reflected, $10 \%$ experienced Lambertian diffuse scattering, and $40 \%$ were absorbed [21].

The electrodes on top of the LED chip were not considered, for the simplicity of the calculation. The emission spectrum of the LED chip had a peak at $460 \mathrm{~nm}$ and followed a Gaussian distribution between $360 \mathrm{~nm}$ and $560 \mathrm{~nm}$ with a FWHM(full width at half maximum) of $50 \mathrm{~nm}$. Alumina, the material used for the substrate and ribs, has a reflectance of $80 \%$. The thickness of the encapsulant, which had a transmittance of $97 \%$, was $1.4 \mathrm{~mm}$. Fresnel reflection was assumed to occur at material interfaces with different refractive indices such as chip/air, chip/encapsulant, and encapsulant/air. For the ray-tracing simulation, 4 million light rays were generated at the bottom of the LED chip and their optical

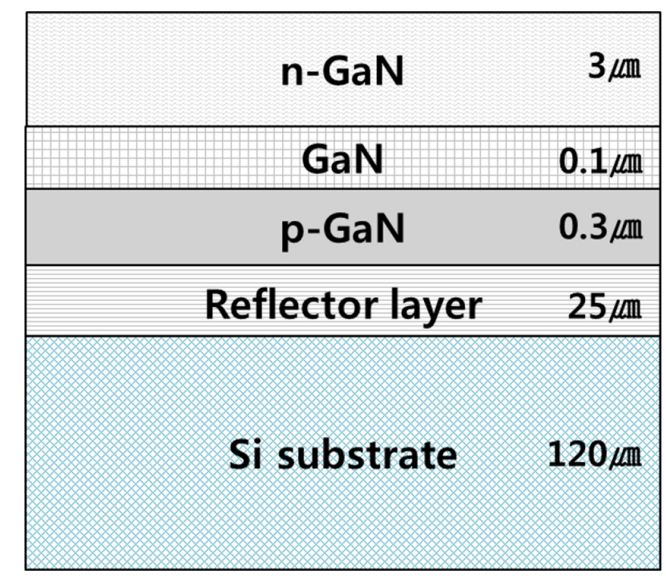

FIG. 3. Schematic drawing of the LED chip consisting of multiple-layers. paths traced using the simulator, which were measured by a detector which was distributed on the inside of the integrating sphere (diameter $1 \mathrm{~m}$ ).

\section{RESULTSAND DISCUSSION}

\subsection{LOP according to the Encapsulant Design (Measured and Simulated)}

Figure 4 shows schematic drawings of five different designs for the encapsulant. In cases (a) and (b), the package was filled with an encapsulant with a refractive index of 1.41 and 1.53 respectively. The thickness of the encapsulant was $1.4 \mathrm{~mm}$. In cases (c), (d), and (e), two different encapsulant materials with refractive indices of 1.41 and 1.53 were stacked in the form of a double layer. The total thickness was $1.4 \mathrm{~mm}$, but the shape of the interface between the stacked layers was different in each case: (c) was flat, (d) was concave, and (e) was convex.

Figure 5 shows the results of the measured LOP using an integrating sphere. The case of the double-layer encapsulant with a convex interface, (e), showed the highest value of $390 \mathrm{~mW}$, which is $13 \%$ higher than the $344 \mathrm{~mW}$ of case (a). To investigate and analyze the change of the LOP according to the design of the encapsulant, a 3-D ray-tracing simulation was conducted.

Figure 6 shows the simulation data of the LOP of the

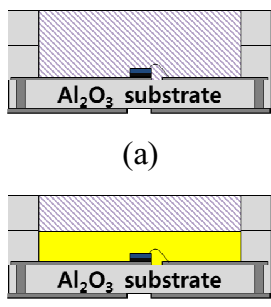

(c)

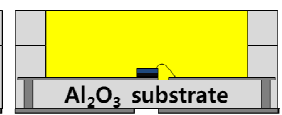

(b)

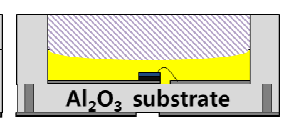

(d)

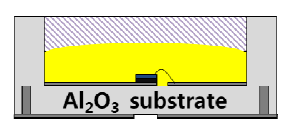

(e)
FIG. 4. Schematic drawings of LED packages with five different encapsulant designs.

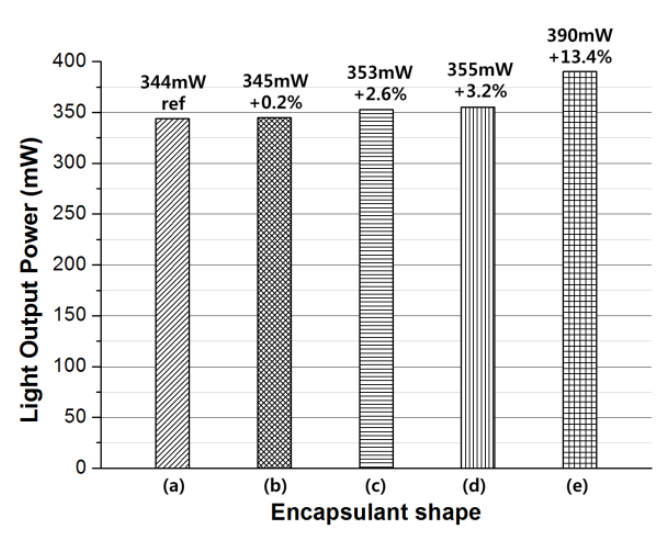

FIG. 5. Measured LOP data for LED packages with the five different encapsulant designs shown in Fig. 4. 
LED package according to the encapsulant design. Figure 7 is a schematic drawing of the curved interface, in which the radius of curvature $R$ was $20 \mathrm{~mm}$ and the thickness of the encapsulant at the center point was fixed at $0.45 \mathrm{~mm}$ and $0.95 \mathrm{~mm}$ respectively for the concave and convex interfaces. We note that in Fig. 6 the double-layer encapsulant with a convex interface, (e), showed the highest value of $399 \mathrm{~mW}$, which was $14.6 \%$ higher compared to that of (a), in good accord with the measured data shown in Fig. 5. From the measured data in Fig. 5 and the simulated data shown in Fig. 6, we know that the double-layer encapsulant with a convex interface, (e), showed the highest LOP.

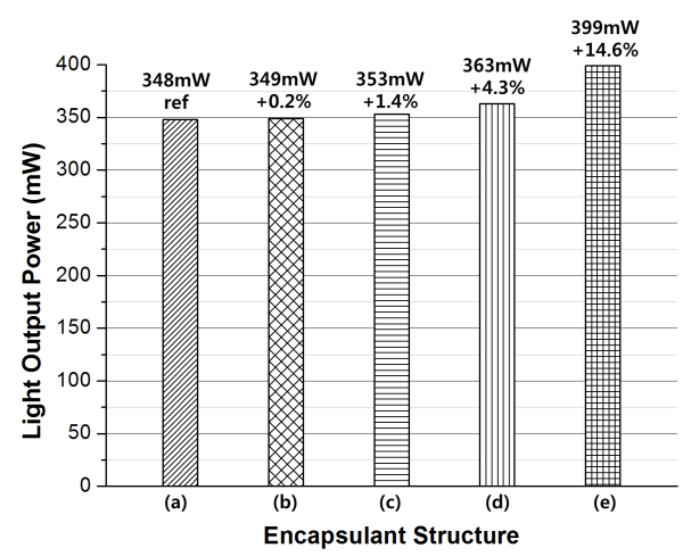

FIG. 6. Simulated LOP data for LED packages with the five different encapsulant designs shown in Fig. 4.

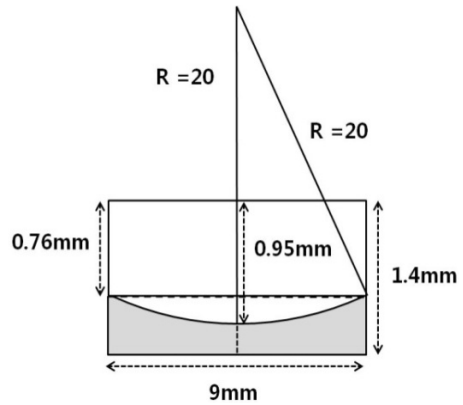

(a)

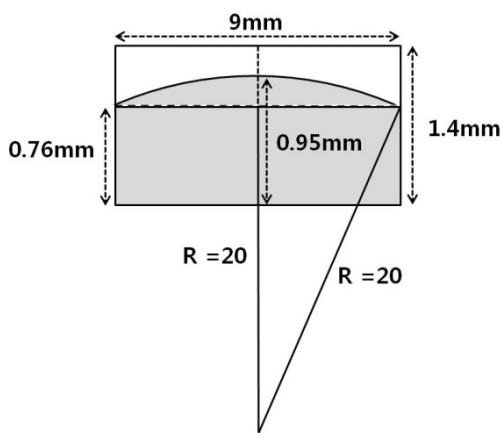

(b)

FIG. 7. Schematic drawing of the curved interface: (a) concave, (b) convex.
We assume that the enhancement is related to the curved interface between the two encapsulant layers with different refractive indices of 1.41 and 1.53 .

To understand the effect of the curved interface of the double-layer encapsulant on the LOP of the LED package, we investigated related factors such as the LEE, the lightescape cone for $\theta_{c}$, and the Fresnel reflection loss based on the ray-tracing simulation.

\subsection{Increase of the Critical Angle $\theta_{c}$ at the Curved Interface}

Figure 8 shows the optical paths of some reflected rays with incident angles beyond the original critical angle $\left(23.18^{\circ}\right)$ at the curved interface, which was investigated using 3-D ray-tracing simulation. In the cases of concave (a) and convex (b) interfaces, critical angle $\theta_{c}$ was seen increase to $23.93^{\circ}$ and $24.72^{\circ}$ respectively. Figure 8 shows that some light rays which were reflected at the curved interface can escape the chip, even though their original incident angle was greater than $23.18^{\circ}$. Since this increase
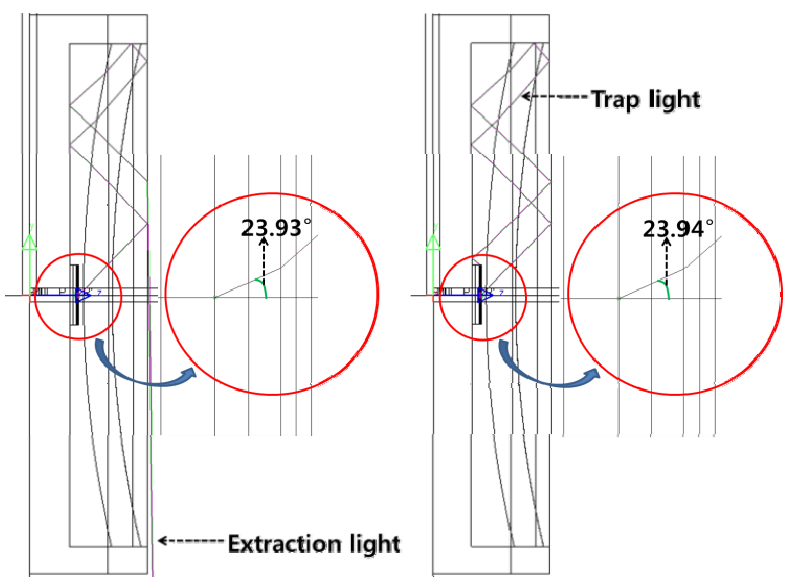

(a)
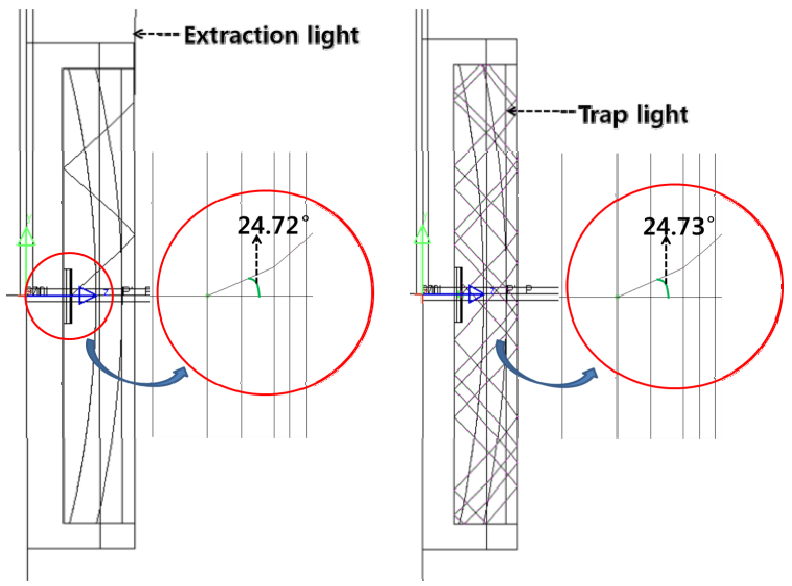

(b)

FIG. 8. Extraction of some reflected rays beyond the original critical angle at the curved interface (3-D ray tracing simulation): (a) concave, (b) convex. 
TABLE 2. Calculation of $\mathrm{LEE}_{\mathrm{ec}}$ using the effective critical angle $\theta_{\mathrm{c}}$ obtained in Fig. 8

\begin{tabular}{|c|c|c|c|}
\hline & & $\theta_{\mathrm{c}}$ & $\mathrm{LEE}_{\mathrm{ec}}$ \\
\hline no encap. & chip $\rightarrow$ air & \multirow{4}{*}{$23.18^{\circ}$} & \multirow{4}{*}{$8.07 \%$} \\
\hline (a) single & chip $\rightarrow$ encap. $(1.41) \rightarrow$ air & & \\
\hline (b) single & chip $\rightarrow$ encap. (1.53) $\rightarrow$ air & & \\
\hline (c) double & $\begin{array}{l}\text { chip } \rightarrow \text { encap. (1.53) } \\
\rightarrow \text { encap. }(1.41) \rightarrow \text { air }\end{array}$ & & \\
\hline (d) concave & $\begin{array}{l}\text { chip } \rightarrow \text { encap. }(1.53) \\
\rightarrow \text { encap. }(1.41) \rightarrow \text { air } \\
{[\text { concave }]}\end{array}$ & $23.51^{\circ}$ & $8.30 \%$ \\
\hline (e) convex & $\begin{array}{l}\text { chip } \rightarrow \text { encap. }(1.53) \\
\rightarrow \text { encap. }(1.41) \rightarrow \text { air } \\
{[\text { convex }]}\end{array}$ & $24.91^{\circ}$ & $9.30 \%$ \\
\hline
\end{tabular}

in $\theta_{c}$ is due to the characteristic of the curved interfaces, we define the increased $\theta_{c}$ at the curved interfaces as the 'effective critical angle'. The basic cause of the effective critical angle can be explained in two ways. First, owing to the curved interface, a normal line at its point of tangency tilts a little, which changes the incident angle and increases $\theta_{c}$ at the curved interface. Secondly, rays reflected at a free surface have a decreased incident angle at the curved interface, which enables some of the reflected rays to escape the chip.

To consider the geometrical factors, we have obtained the effective critical angle $\theta_{c}$ at 9 different positions at the interface and averaged them, to obtain $23.51^{\circ}$ and $24.91^{\circ}$ respectively. We have used these average values of the effective critical angle for Table 2.

Table 2 shows that the LEE ec values of (a), (b), and (c) are same as in the case of no encapsulation, because the same $\theta_{c}$ applies. It should be noted that $\theta_{c}$ mentioned in Table 2 is the critical angle at the free surface, which depends on the refractive indices of the chip and air only. In other words, the intermediate media between the LED chip and the air do not affect the critical angle at the free surface, which is an optical characteristic for a multilayered structure with flat interfaces.

The effective critical angles increased to $23.51^{\circ}$ and $24.91^{\circ}$, respectively for (d) and (e), which resulted in $\mathrm{LEE}_{\mathrm{ec}}$ values of $8.3 \%$ and $9.3 \%$ respectively, which were higher than in other cases (8.07\%).

\subsection{Fresnel Reflection Loss at the Curved Interface}

In general, when a wave reaches the boundary between two media with different refractive indices, one part of the wave is reflected and the other is transmitted, with the sum of the energies of these two waves equal to that of the original wave. It is well known that the light is split into $s$ and $p$ polarizations. The reflective coefficients for $s$-polarization and $p$-polarization are described as follows:

$$
\begin{aligned}
& r_{s}=\frac{\cos \theta-\sqrt{n^{2}-\sin ^{2} \theta}}{\cos \theta+\sqrt{n^{2}-\sin ^{2} \theta}} \\
& r_{D}=-\frac{n^{2} \cos \theta-\sqrt{n^{2}-\sin ^{2} \theta}}{n^{2} \cos \theta+\sqrt{n^{2}-\sin ^{2} \theta}}
\end{aligned}
$$

where $n$ is defined as $n_{\mathrm{t}} / n_{\mathrm{i}}$ in which $n_{\mathrm{t}}$ and $n_{\mathrm{i}}$ are the refractive indices of the transmitted and incident media respectively. The reflectances for $s$ - and $p$-polarizations can be obtained from the following relationships:

$$
\begin{aligned}
& R_{s}=\left|r_{s}\right|^{2} \\
& R_{D}=\left|r_{D}\right|^{2}
\end{aligned}
$$

On the other hand, when multiple interfaces are involved the reflectances for $s$ - and $p$-polarizations can be obtained from the following relationships:

$$
\begin{aligned}
& R_{p}=\left[1-\left(1-R_{p_{1}}\right)\left(1-R_{p_{2}}\right)\left(1-R_{p_{3}}\right)\right] \\
& R_{s}=\left[1-\left(1-R_{s 1}\right)\left(1-R_{s 2}\right)\left(1-R_{s 3}\right)\right]
\end{aligned}
$$

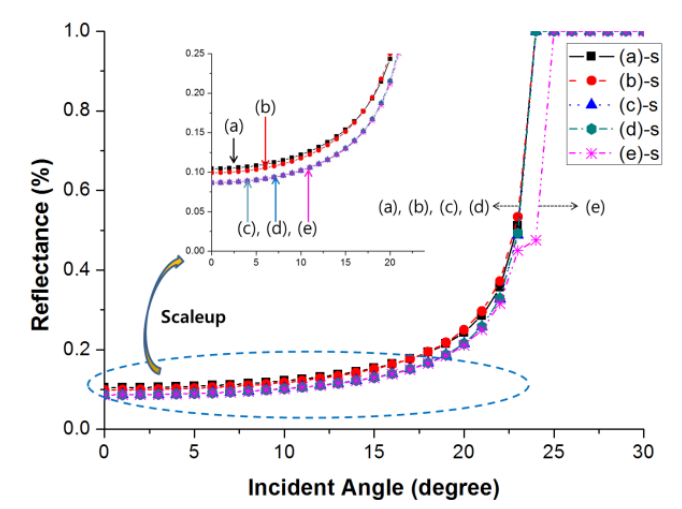

(a)

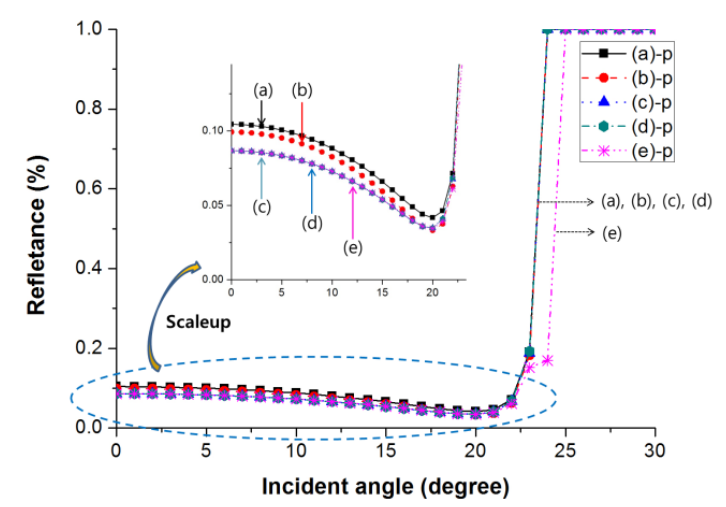

(b)

FIG. 9. Fresnel reflection loss with the incident angle: (a) $s$-polarization, (b) $p$-polarization. 
where $n$ is the number of the interface and $R_{n}$ is the reflectance at the $n$th interface.

Figure 9 shows the Fresnel reflectance according to the incident angle. We input the incident angle into Eqs. (3) and (4) with an increment of $1^{\circ}$ in the range from $0^{\circ}$ to $\theta_{c}$, which was converted to reflectance using Eqs. (5) and (6). The Fresnel reflection loss was determined using Eqs. (7) and (8) with the reflectance values at every interface. In the cases of the curved interfaces, (d) and (e), the effective $\theta_{c}$ was obtained using a 3-D ray-tracing simulation, as mentioned above.

Two different features were found in Fig. 9, according to the range of the incident angle. For the range between $0^{\circ}$ and $30^{\circ}$, both $s$ - and $p$-polarizations showed that the Fresnel reflectance was smaller for the double-layer encapsulant in (c), (d), and (e), compared to the single layer of (a) and (b). We included the insets in Fig. 9 to contrast the relative values close up, in which it could be seen that the Fresnel loss was smaller for the multilayered structure than the single-layer structure. This can be explained by Eq. (7) and (8), in which the reflectance decreases with the number of the interfaces when a graded refractive index is used for each layer. On the other hand, for the range between $20^{\circ}$ and $25^{\circ}$, both $s$ - and p-polarizations showed that the Fresnel reflectance was the smallest for a doublelayer encapsulant with a convex interface, (e). These two features were assumed to be the reason why we obtained the highest LOP for the encapsulant design (e) in Fig. 5 and Fig. 6. For a quantitative analysis, we integrated the curves of the Fresnel reflectance shown in Fig. 9, as described in Table 3.

In Table 3 we determined the total LEE by multiplying the transmittance and the $\mathrm{LEE}_{\mathrm{ec}}$. In Table 3 the total reflectance of the rays passing through the encapsulant was defined as

$$
R=\frac{R_{s}+R_{p}}{2}
$$

TABLE 3. Determination of the total LEE by multiplying the transmittance and the $\mathrm{LEE}_{\mathrm{ec}}$

\begin{tabular}{l|c|c|c|c|c|c}
\hline \hline Encapsulation designs & $\begin{array}{c}\mathrm{R}_{\mathrm{s}} \\
(\%)\end{array}$ & $\begin{array}{c}\mathrm{R}_{\mathrm{p}} \\
(\%)\end{array}$ & $\begin{array}{c}\mathrm{R} \\
(\%)\end{array}$ & $\begin{array}{c}\mathrm{T} \\
(\%)\end{array}$ & $\begin{array}{c}\mathrm{LEE}_{\mathrm{ec}} \\
(\%)\end{array}$ & $\begin{array}{c}\mathrm{LEE}_{\text {total }} \\
(\%)\end{array}$ \\
\hline No encapsulation & 31.35 & 15.85 & 23.60 & 76.40 & 8.07 & 6.17 \\
\hline $\begin{array}{l}\text { (a) Single layer with } \\
\mathrm{n}=1.41\end{array}$ & 20.78 & 12.57 & 16.68 & 83.32 & 8.07 & 6.72 \\
\hline $\begin{array}{l}\text { (b) Single layer with } \\
\mathrm{n}=1.53\end{array}$ & 20.84 & 12.01 & 16.42 & 83.58 & 8.07 & 6.74 \\
\hline $\begin{array}{l}\text { (c) } 1.53 / 1.41 \text { with } \\
\text { flat interface }\end{array}$ & 18.78 & 11.38 & 15.08 & 84.92 & 8.07 & 6.85 \\
\hline $\begin{array}{l}\text { (d) } 1.53 / 1.41 \text { with } \\
\text { concave interface }\end{array}$ & 18.65 & 11.23 & 14.94 & 85.16 & 8.30 & 7.07 \\
\hline $\begin{array}{l}\text { (e) } 1.53 / 1.41 \text { with } \\
\text { convex interface }\end{array}$ & 16.43 & 9.32 & 12.86 & 87.14 & 9.30 & 8.08 \\
\hline
\end{tabular}

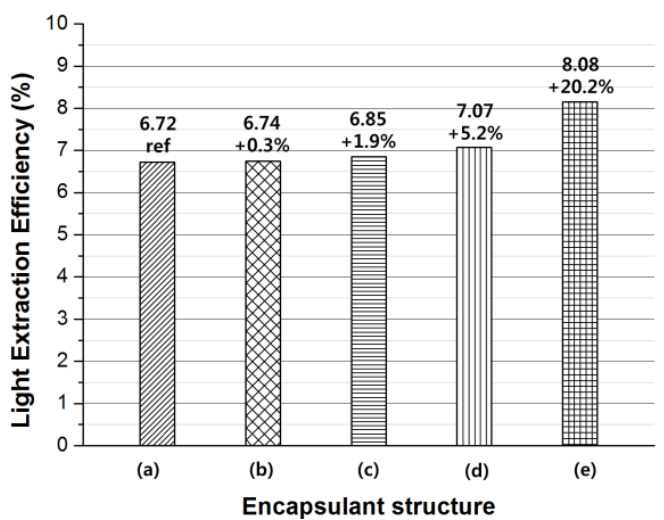

FIG. 10. LEE $_{\text {tot }}$ of the LED packages with the five different encapsulant designs shown in Fig. 4.

Therefore, the total transmittance of the rays passing through the encapsulant was defined as

$$
T=1-R
$$

It can be seen in Table 3 that $T$ is highest for a doublelayer encapsulant with a convex interface, (e), at $87.14 \%$.

In Table 3 we define the total light extraction efficiency $\mathrm{LEE}_{\text {tot }}$ as follows:

$$
L E E_{t o t}=L E E_{e c} \times T
$$

where $\mathrm{LEE}_{\mathrm{ec}}$ is the light extraction efficiency determined by the light-escape cone using Eq. (2), and $T$ is the total transmittance of the rays passing through the encapsulant using Eq. (10). It can be seen in Table 3 that $\mathrm{LEE}_{\text {tot }}$ is the highest for (e) at $8.08 \%$, which is $31 \%$ higher than in the case of no encapsulation and $20.2 \%$ higher than in (a). Figure 10 compares the $\mathrm{LEE}_{\text {tot }}$ values for the five different encapsulant designs of Fig. 4, which is in good agreement with the measured results (see Fig. 5) and simulated values (see Fig. 6). These results demonstrate convincingly that our assumption using the light-escape cone and the Fresnel reflection loss at the curved interface between the two different encapsulant materials in this study explains well the $20.2 \%$ higher LOP for the double-layer encapsulation with a convex interface in (e).

\section{CONCLUSION}

By experiment and 3-D ray-tracing simulation, we have shown that the LOP of an LED package was most effectively enhanced by structure (e) using a double-layered convex-interface encapsulant, resulting in a $13.4 \%$ increase compared to a single-layer encapsulant structure (a). The enhancement mechanism was explained in two ways.

First, from the simulation it could be seen that the critical angle was the highest at $24.91^{\circ}$ for (e), compared 
to $23.18^{\circ}$ for (a), which was explained by the concept of the effective critical angle at a curved surface. Second, total transmittance $T$ of the rays passing through the encapsulant was the highest at $87.14 \%$ for (e), compared to $83.32 \%$ for (a), which was explained by the decrease of the Fresnel reflection loss due to the double-layered structure. Conclusively, it was seen that the double-layered convex-interface encapsulant was most effective at both increasing the critical angle and decreasing the Fresnel reflection loss.

\section{ACKNOWLEDGMENT}

This research was financially supported by the Ministry of Education, Science and Technology (MEST) of the Korean government, and the National Research Foundation of Korea (NRF) through the Human Resource Training Project for Regional Innovation (no. 2011H8A2003348). The authors gratefully acknowledge this support.

\section{REFERENCES}

1. T. X. Lee, K. F. Gao, W. T. Chien, and C. C. Sun, "Light extraction analysis of GaN-based light-emitting diodes with surface texture and/or patterned substrate," Opt. Express 15, 6670-6676 (2007).

2. J. H. Lee, J. T. Oh, S. B. Choi, Y. C. Kim, H. I. Cho, and J. H. Lee, "Enhancement of InGaN-based vertical LED with concavely patterned surface using patterned sapphire substrate," IEEE Photon. Tech. Lett. 20, 345-347 (2008).

3. Y. K. Ee, P. Kumnorkaew, R. A. Arif, H. Tong, J. F. Gilchrist, and N. Tansu, "Light extraction efficiency enhancement of InGaN quantum wells light-emitting diodes with polydimethylsiloxane concave microstructures," Opt. Express 17, 13747-13757 (2009).

4. Y. S. Park, H. G. Lee, C. M. Yang, D. S. Kim, J. H. Bae, S. J. Cho, J. H. Lee, and I. M. Kang, "Fabrication and characterization of a GaN light-emitting diode (LED) with a centered island cathode," J. Opt. Soc. Korea 16, 349-353 (2012).

5. A. I. Zhmakin, "Enhancement of light extraction from light emitting diodes," Physics Reports 498, 189-241 (2011).

6. X. H. Wang, W. Y. Fu, P. T. Lai, and H. W. Choi, "Evaluation of InGaN/GaN light-emitting diodes of circular geometry," Opt. Express 17, 22311-22319 (2009).

7. M. R. Krames, O. B. Shchekin, R. M. Mach, G. O. Mueller, L. Zhou, G. Harbers, and M. G. Craford, "Status and future of high-power light-emitting diodes for solid-state lighting," IEEE J. Display Technol. 3, 160-175 (2007).

8. J. H. Kang, J. H. Ryu, H. K. Kim, H. Y. Kim, N. Han, M. S. Lee, Y. J. Park, P. Uthirakumar, V. V. Lysak, and C. H. Hong, "Enhancement of light output power in GaN-based light-emitting diodes using indium tin oxide films with nanoporous structures," Thin Solid Films 520, 437-441 (2011).
9. X. H. Li, R. Song, Y. K. Ee, P. Kumnorkaew, J. F. Gilchrist, and N. Tansu, "Light extraction efficiency and radiation patterns of III-Nitride light-emitting diodes with colloidal microlens arrays with various aspect ratios," IEEE Photonics Journal 3, 489-499 (2011).

10. J. Y. Cho, K. J. Byeon, H. G. Park, J. S. Kim, H. S. Kim, and H. Lee, "Improvement of photon extraction efficiency of GaN-based LED using micro and nano complex polymer structures," Nanoscale Research Letters 6, 578, 1-6 (2011).

11. S. H. Kim, K. D. Lee, J. Y. Kim, M. K. Kwon, and S. J. Park, "Fabrication of photonic crystal structures on light emitting diodes by nanoimprint lithography," Nanotechnology 18, 055306 (2007).

12. J. K. Kim, S. Chhajed, M. F. Schubert, E. F. Schubert, A. J. Fischer, M. H. Crawford, J. H. Cho, H. S. Kim, and C. S. Sone, "Light-extraction enhancement of GaInN light-emitting diodes by graded-refractive-index indium tin oxide anti-reflection contact," Adv. Meter. 20, 801-804 (2008).

13. Y. M. Song, E. S. Choi, J. S. Yu, and Y. T. Lee, "Light-extraction enhancement of red AlGaInP light-emitting diodes with antireflective subwavelength structures," Opt. Express 17, 20991-20997 (2009).

14. M. T. Lin, S. P. Ying, M. Y. Lin, K. Y. Tai, S. C. Tai, C. H. Liu, J. C. Chen, and C. C. Sun, "Design of the ring remote phosphor structure for phosphor-converted white-lightemitting diodes," Jap. J. Appl. Phys. 49, 072101 (2010).

15. B. T. Liu, S. J. Tang, Y. Y. Yu, and S. H. Lin, "Highrefractive-index polymer/inorganic hybrid films containing high $\mathrm{TiO}_{2}$ contents," Colloids and Surfaces A: Physicochem. Eng. Aspects 377, 138-143 (2011).

16. Y. H. Lin, J. P. You, Y. C. Lin, N. T. Tran, and F. G. Shi, "Development of high-performance optical silicone for the packaging of high-power LEDs," IEEE Trans. Compon. Packag. Manuf. Technol. 33, 761-766 (2010).

17. N. Gao, W. Q. Liu, S. Q. Ma, C. Tang, and Z. L. Yan, "Cycloaliphatic epoxy resin modified by two kinds of oligofluorosiloxanes for potential application in light-emitting diode (LED) encapsulation," J. Polym. Res. 19, 9923 (2012).

18. Y. Zhou, N. Tran, Y. C. Lin, Y. He, and F. G. Shi, "One-component, low-temperature, and fast cure epoxy encapsulant with high refractive index for LED applications," IEEE Trans. Compon. Packag. Manuf. Technol. 31, 484-489 (2008).

19. M. Ma, F. W. Mont, X. Yan, J. H. Cho, E. F. Schubert, G. B. Kim, and C. S. Sone, "Effects of the refractive index of the encapsulant on the light-extraction efficiency of light-emitting diodes," Opt. Express 19, A1135-A1140 (2011).

20. R. Hu, X. Luo, and S. Liu, "Study on the optical properties of conformal coating light-emitting diode by Monte Carlo simulation," IEEE Photon. Technol. Lett. 23, 1673-1675 (2011).

21. Z. Liu, K. Wang, X. Luo, and S. Liu, "Precise optical modeling of blue light-emitting diodes by Monte Carlo ray-tracing," Opt. Express 18, 9398-9412 (2010). 